\title{
LOCALIZATIONS OF INJECTIVE MODULES
}

\author{
by K. R. GOODEARL and D. A. JORDAN
}

(Received 9th April 1984)

The question of whether an injective module $E$ over a noncommutative noetherian ring $R$ remains injective after localization with respect to a denominator set $X \subseteq R$ is addressed. (For a commutative noetherian ring, the answer is well-known to be positive.) Injectivity of the localization $E\left[X^{-1}\right]$ is obtained provided either $R$ is fully bounded (a result of $\mathrm{K}$. A. Brown) or $X$ consists of regular normalizing elements. In general, $E\left[X^{-1}\right]$ need not be injective, and examples are constructed. For each positive integer $n$, there exists a simple noetherian domain $R$ with Krull and global dimension $n+1$, a left and right denominator set $X$ in $R$, and an injective right $R$-module $E$ such that $E\left[X^{-1}\right]$ has injective dimension $n$; moreover, $E$ is the injective hull of a simple module.

The research of the first author was partially supported by a National Science Foundation grant. The work was done while the second author was visiting the Mathematics Department of the University of Utah, and he wishes to thank that department for their hospitality.

\section{Preservation of injectivity}

Given a right or left denominator set $X$ in a ring $R$, we write $t_{X}(E)$ and $E\left[X^{-1}\right]$ for the $X$-torsion submodule of an $R$-module $E$ and the $X$-localization of $E$. Assuming that $E$ is an injective $R$-module, we consider the problem of deciding whether $E\left[X^{-1}\right]$ must be an injective $R\left[X^{-1}\right]$-module. Recall that $E\left[X^{-1}\right]$ is injective as an $R\left[X^{-1}\right]$-module if and only if it is injective as an $R$-module [11, Exercise 12, p. 62].

In case $R$ is commutative noetherian, or, more generally, if $R$ is noetherian and $X$ is central, $E\left[X^{-1}\right]$ must be injective [2, Lemma 1.2]. However, for $R$ commutative but not noetherian, $E\left[X^{-1}\right]$ need not be injective [5, Theorems 25,28$]$. In the noncommutative fully bounded noetherian case, $K$. A. Brown has proved the following positive result, and we thank him for communicating it for presentation here.

Theorem 1.1. (Brown) Let $R$ be a right and left noetherian right fully bounded ring, let $X$ be a right and left denominator set in $R$, and let $E$ be an injective right $R$-module. Then $E\left[X^{-1}\right]$ is an injective right $R\left[X^{-1}\right]$-module.

Proof. It suffices to consider the case that $E$ is indecomposable. If $E$ is $X$-torsion, then $E\left[X^{-1}\right]=0$, while if $E$ is $X$-torsion-free, then $E\left[X^{-1}\right]=E$; in either case, $E\left[X^{-1}\right]$ is injective. Hence, it is enough to show that $E$ is either $X$-torsion or $X$-torsion-free. 
Let $T=t_{X}(E)$ and suppose that $T \neq 0$ and $T \neq E$. Since $T \neq E$ we may choose a finitely generated submodule $B$ of $E$ such that $B \nsubseteq T$ and $\operatorname{ann}(B)$ is maximal among the annihilators of such submodules. Set $C=B \cap T$ and $Q=\operatorname{ann}(B / C)$; thus $B Q \subseteq T$. By the $H$-condition [3, Theorem 7.8], the annihilator of $B Q$ equals the annihilator of some finite subset of $B Q$, and hence there exists $x \in X$ such that $B Q x=0$. It follows that $Q$ is prime, for if $I$ and $J$ are ideals of $R$ such that $I \nsubseteq Q$ and $I J \subseteq Q$, then $B I J x=0$ and $J x$ is contained in $\operatorname{ann}(B I)$, which, by maximality, equals ann $(B)$; then $B J x=0$ and so $B J \subseteq \mathrm{T}$, whence $J \subseteq Q$.

The image $\bar{X}$ of $X$ in $R / \operatorname{ann}(B)$ is a left Ore set, and, because $R / \operatorname{ann}(B)$ is left noetherian, $\bar{X}$ must be a left denominator set [11, Proposition II.1.5]. Hence, since $Q x \subseteq \operatorname{ann}(B)$, there exists $y \in X$ such that $y Q \subseteq$ ann $(B)$, and $B y Q=0$. But $B y R \nsubseteq T$ because $B$ is not $X$-torsion, and so, by maximality of $\operatorname{ann}(B)$, we obtain $\operatorname{ann}(B y R)=$ $\operatorname{ann}(B)$. Thus $Q \subseteq \operatorname{ann}(B)$, and so $Q=\operatorname{ann}(B)$.

By the $H$-condition, $R / Q$ embeds in a finite direct sum of copies of $B$. Let $U$ be a uniform right ideal of $R / Q$. There is a finite set of homomorphisms $U \rightarrow B$ whose kernels intersect to zero, and hence one of these maps is injective, so that $B$ has a submodule $D$ isomorphic to $U$. Since $E$ is indecomposable, $D \cap T \neq 0$. It follows that $t_{X}(U) \neq 0$ and hence that $t_{X}(R / Q) \neq 0$.

But $t_{X}(R / Q)$ is an ideal of $R / Q$ and so, since $Q$ is prime, $t_{X}(R / Q)$ must contain a regular element $c$. As $c y=0$ for some $y \in X$, we conclude that $y \in Q$ and $B y=0$, so that $B$ is $X$-torsion. This contradicts the choice of $B$.

Therefore $E$ is either $X$-torsion or $X$-torsion-free, as desired.

Another case in which localizations of injective modules are injective is that of a denominator set consisting of regular normalizing elements. (Recall that a normalizing element in a ring $R$ is any element $c \in R$ such that $c R=R c$.)

Lemma 1.2. Let $x$ be a regular normalizing element in a ring $R$, let $E$ be an injective right $R$-module, and set $A=\{a \in E \mid a x=0\}$. Then $E / A$ is an injective right $R$-module.

Proof. That $A$ is a submodule of $E$ follows because $x$ is a normalizing element. Since $x$ is regular, there exist homomorphisms $x R \rightarrow E$ sending $x$ to any element of $E$; by injectivity, $E x=E$.

For any $r \in R$, there is a unique element $\varphi(r) \in R$ such that $x r=\varphi(r) x$. Observe that $\varphi$ is a ring automorphism of $R$.

Now right multiplication by $x$ defines an abelian group epimorphism $E \rightarrow E$ with kernel $A$. This induces an abelian group isomorphism $f: E / A \rightarrow E$ such that $f q(b)=b x$ for all $b \in E$, where $q: E \rightarrow E / A$ is the quotient map. For all $b \in E$ and $r \in R$, we compute that

$$
f(q(b) \varphi(r))=f q(b \varphi(r))=b \varphi(r) x=b x r=f q(b) r .
$$

Hence, $f(c \varphi(r))=f(c) r$ for all $c \in E / A$ and all $r \in R$.

Let $J$ be a right ideal of $R$ and $g: J \rightarrow E / A$ an $R$-module homomorphism. Then $\varphi^{-1}(J)$ is a right ideal of $R$ and $f g \varphi$ is a group homomorphism from $\varphi^{-1}(J)$ to $E$. For all $t \in J$ and $r \in R$, we check that

$$
f g \varphi\left(\varphi^{-1}(t) r\right)=f g(t \varphi(r))=f(g(t) \varphi(r))=f g(t) r=f g \varphi\left(\varphi^{-1}(t)\right) r,
$$


so that $f g \varphi$ is an $R$-module homomorphism. Hence, there exists $b \in E$ such that $f g \varphi(s)=b s$ for all $s \in \varphi^{-1}(J)$. Also, $b=f(c)$ for some $c \in E / A$. For all $t \in J$, we have

$$
f g(t)=f g \varphi\left(\varphi^{-1}(t)\right)=b \varphi^{-1}(t)=f(c) \varphi^{-1}(t)=f(c t),
$$

whence $g(t)=c t$.

Therefore $E / A$ is injective.

Theorem 1.3. Let $R$ be a right noetherian ring, let $X$ be a right denominator set of regular normalizing elements of $R$, and let $E$ be an injective right $R$-module. Then $E\left[X^{-1}\right]$ is an injective right $R\left[X^{-1}\right]$-module.

Proof. As $X$ consists of regular elements, $E$ is $X$-divisible. Hence, $E\left[X^{-1}\right]=E / A$, where $A=t_{X}(E)$. Set $A_{x}=\{a \in E \mid a x=0\}$ for all $x \in X$, and note that $A$ is the union of the submodules $A_{x}$. Given any $x, y \in X$, there exist $r \in R$ and $z \in X$ such that $x r=y z$, whence $y z \in X$ and $A_{x} \cup A_{y} \subseteq A_{y z}$. Thus $A$ is a directed union of the $A_{x}$.

Now $E\left[X^{-1}\right]$ is isomorphic to a direct limit of the modules $E / A_{x}$, each of which is injective by Lemma 1.2. Since $R$ is right noetherian, $E\left[X^{-1}\right]$ is injective as a right $R$ module, and therefore also as a right $R\left[X^{-1}\right]$-module.

\section{Loss of injectivity}

An example of an injective module which has a localization that is not injective is constructed in this section. As the coefficient ring for this example is a differential operator ring, we recall some of the terminology associated with such rings.

The term differential ring is used to denote a ring (associative, with unit) equipped with a specified derivation. For ease of notation, all derivations in this paper will be denoted $\delta$. A differential ring which is also a domain, or a field, is called a differential domain, or a differential field. The subring of constants of a differential ring $R$ is the set $\{r \in R \mid \delta(r)=0\}$, which, as is readily seen, is a subring of $R$. In case $R$ is a differential field, its subring of constants is a subfield of $R$, and so is called the subfield of constants.

The formal linear differential operator ring associated with a differential ring $R$, denoted $R[\theta ; \delta]$, is a ring which additively is the abelian group of all polynomials over $R$ in an indeterminate $\theta$, and in which multiplication is induced from the multiplication in $R$ via the rule $\theta r=r \theta+\delta(r)$, for all $r \in R$. If $T=R[\theta ; \delta]$, then $R$ can be viewed as a left $T$-module by extending the left $R$-module multiplication of ${ }_{R} R$ to a left $T$-module multiplication o under which $\theta \circ r=\delta(r)$ for all $r \in R$. (The module ${ }_{r} R$ constructed in this fashion is isomorphic to $T / T \theta$.) Similarly, if $S$ is a differential ring extension of $R$, then $S$ can be viewed as a left $S[\theta ; \delta]$-module and hence as a left $T$-module.

If $R$ is a commutative differential domain, it will be convenient to obtain the injective hull $E\left({ }_{T} R\right)$ as a $T$-submodule of a suitable differential field extension of the quotient field of $R$. This differential field extension is constructed so as to contain solutions for all linear differential equations, as follows.

Proposition 2.1. Given a differential field $F$, there exists a differential field extension $\vec{F}$ of $F$ such that every nonhomogeneous linear differential equation over $\bar{F}$ has a solution in $\bar{F}$. 
Proof. It is enough to show that any differential field $F_{1}$ has a differential field extension $\sigma\left(F_{1}\right)$ such that every nonhomogeneous linear differential equation over $F_{1}$ has a solution in $\sigma\left(F_{1}\right)$, since then the union of the differential fields

$$
F \subseteq \sigma(F) \subseteq \sigma^{2}(F) \subseteq \ldots
$$

can be taken for $\bar{F}$. Such a differential field extension $\sigma\left(F_{1}\right)$ can be constructed by transfinite induction, provided it can be shown that any nonhomogeneous linear differential equation over a differential field $F_{2}$ has a solution in a differential field extension of $F_{2}$.

Hence, consider a differential equation

$$
\alpha_{n} \delta^{n}(x)+\alpha_{n-1} \delta^{n-1}(x)+\cdots+\alpha_{1} \delta(x)+\alpha_{0} x=\beta
$$

where $\alpha_{i}, \beta \in F_{2}$ and $\alpha_{n} \neq 0$. There is no loss of generality in assuming that $\alpha_{n}=1$, for otherwise we can multiply throughout $\left({ }^{*}\right)$ by $\alpha_{n}^{-1}$. Set $F_{3}$ equal to a rational function field $F_{2}\left(y_{0}, y_{1}, \ldots, y_{n-1}\right)$ where the $y_{j}$ are algebraically independent over $F_{2}$, and extend $\delta$ to a derivation of $F_{3}$ by the rules $\delta\left(y_{j}\right)=y_{j+1}$ for $j=0, \ldots, n-2$, while

$$
\delta\left(y_{n-1}\right)=\beta-\alpha_{n-1} y_{n-1}-\ldots-\alpha_{1} y_{1}-\alpha_{0} y_{0}
$$

Then $y_{0}$ is a solution of $(*)$ in the differential field extension $F_{3}$ of $F_{2}$.

The solvability of nonhomogeneous linear differential equations obtained in Proposition 2.1 says precisely that $\bar{F}$ is divisible as a left $\bar{F}[\theta ; \delta]$-module. We shall use this in the situation where $F$ is the quotient field of a commutative differential domain $R$. (The derivation on $R$ extends uniquely to a derivation on $F$ by the quotient rule.) Of course $\bar{F}$ is also divisible when viewed as an $F[\theta ; \delta]$-module or as an $R[\theta ; \delta]$-module.

Proposition 2.2. Let $R$ be a commutative differential domain, $F$ its quotient field, and $\bar{F}$ as in Proposition 2.1. Set $T=R[\theta ; \delta]$.

(i) The T-module ${ }_{T} \bar{F}$ is injective, and the submodule ${ }_{T} F$ is an essential extension of ${ }_{T} R$. Consequently, the injective hull of ${ }_{T} R$ can be identified with a T-submodule of $\bar{F}$ containing $F$.

(ii) For any left denominator set $X$ in $T$, the localization $\bar{F}\left[X^{-1}\right]$ of ${ }_{T} \bar{F}$ equals $\bar{F} / t_{X}(\bar{F})$.

Proof. (i) Let $U$ be the ring $F[\theta ; \delta]$. Since $U$ is a left principal ideal domain and $\bar{F}$ is a divisible $U$-module, $\bar{F}$ is an injective $U$-module [10, Theorem 2.8]. Since $U$ is flat as a right $T$-module (e.g., [6, Lemma 7]), it follows that $\bar{F}$ is an injective left $T$-module [8, Theorem IV.12.5]. That ${ }_{T} R$ is essential in ${ }_{T} F$ is clear.

(ii) This is immediate from the divisibility of $\bar{F}$ as a left $T$-module.

Continuing with the notation of Proposition 2.2 , let $X$ be a left denominator set in $T$ such that $t_{X}(F)=R$. Our first aim is to establish sufficient conditions for the natural map from $F / R$ to $\bar{F} / t_{X}(\bar{F})=\bar{F}\left[X^{-, 1}\right]$ to be a split $R$-module monomorphism and hence for $F / R$ to be isomorphic to an $R$-module direct summand of $E\left[X^{-1}\right]$, where $E$ is the injective hull of ${ }_{T} R$. (See Proposition 2.7.) 
Recall that a differential ring $R$ is $\delta$-simple provided $R$ is nonzero and the only $\delta$ ideals (that is, $\delta$-invariant ideals) of $R$ are 0 and $R$.

Proposition 2.3. Let $R$ be a $\delta$-simple differential ring such that the subring $K$ of constants of $R$ is a field. Let $S$ be a simple $K$-algebra, and extend $\delta$ to a derivation on $R \otimes_{K} S$ so that $\delta(r \otimes s)=\delta(r) \otimes s$ for all $r \in R$ and $s \in S$. Then $R \otimes_{K} S$ is $\delta$-simple.

Proof. Let $I$ be a nonzero $\delta$-ideal of $R \bigotimes_{K} S$, and choose a nonzero element

$$
x=\left(r_{1} \otimes s_{1}\right)+\cdots+\left(r_{n} \otimes s_{n}\right) \in I
$$

with $n$ minimal. By the minimality of $n$, the $s_{i}$ are linearly independent over $K$ and each $r_{i} \neq 0$.

As $R$ is $\delta$-simple, $\sum_{j \geq 0} R \delta^{j}\left(r_{1}\right) R=R$ and hence

$$
\sum_{j=0}^{p} \sum_{k=0}^{q} a_{j k} \delta^{j}\left(r_{1}\right) b_{j k}=1
$$

for some elements $a_{j k}, b_{j k} \in R$. Set

$$
y=\sum_{j=0}^{p} \sum_{k=0}^{q}\left(a_{j k} \otimes 1\right) \delta^{j}(x)\left(b_{j k} \otimes 1\right) .
$$

Then $y \in I$ and

$$
y=\left(1 \otimes s_{1}\right)+\left(r_{2}^{\prime} \otimes s_{2}\right)+\cdots+\left(r_{n}^{\prime} \otimes s_{n}\right)
$$

for some elements $r_{j}^{\prime} \in R$. Note that $y \neq 0$ (because the $s_{i}$ are linearly independent). Now

$$
\left(\delta\left(r_{2}^{\prime}\right) \otimes s_{2}\right)+\cdots+\left(\delta\left(r_{n}^{\prime}\right) \otimes s_{n}\right)=\delta(y) \in I .
$$

By the minimality of $n$ and the linear independence of $s_{2}, \ldots, s_{n}$, it follows that $\delta\left(r_{i}^{\prime}\right)=0$ and so $r_{i}^{\prime} \in K$, for $i=2, \ldots, n$. But then

$$
y=1 \otimes\left(s_{1}+r_{2}^{\prime} s_{2}+\cdots+r_{n}^{\prime} s_{n}\right) .
$$

Thus $n=1$ and $y=1 \otimes s_{1}$. By the simplicity of $S$, we have $S s_{1} S=S$, from which we conclude that

$$
1 \otimes 1 \in 1 \otimes\left(S s_{1} S\right) \subseteq\left(R \otimes_{K} S\right) y\left(R \otimes_{K} S\right) \subseteq I
$$

and hence that $I=R \otimes_{\mathrm{K}} S$.

Corollary 2.4. Let $F \subseteq \vec{F}$ be differential fields with subfields $K$ and $\vec{K}$ of constants. Then the multiplication map $\mu$ from $F \bigotimes_{K} \bar{K}$ to $\bar{F}$ is injective, and hence every $K$-linearly independent subset of $F$ is also $\bar{K}$-linearly independent. 
Proof. Extend $\delta$ to a derivation on $F \bigotimes_{K} \bar{K}$ so that $\delta(x \otimes y)=\delta(x) \otimes y$ for all $x \in F$ and $y \in \bar{K}$. Since $F$ is $\delta$-simple, Proposition 2.3 shows that $\mathrm{F}()_{K} \bar{K}$ is $\delta$-simple. It is easy to check that $\mu$ is a nonzero differential ring homomorphism, and hence that $\operatorname{ker}(\mu)$ is a proper $\delta$-ideal of $F \otimes_{K} \vec{K}$. Thus $\operatorname{ker}(\mu)=0$ and $\mu$ is injective. The second conclusion follows immediately.

Proposition 2.5. Let $F \subseteq \bar{F}$ be differential fields with subfields $K$ and $\bar{K}$ of constants. Let $R$ be a differential subring of $F$ and set $\bar{R}=R \bar{K}$. Then the R-module homomorphism $j: F / R \rightarrow \bar{F} / \bar{R}$ induced by the inclusion map $F \rightarrow \bar{F}$ is a split monomorphism.

Proof. The map $j$ can be factorized as follows:

$$
F / R \stackrel{f}{\rightarrow}(F / R) \bigotimes_{K} K \stackrel{1 \otimes g}{\rightarrow}(F / R) \bigotimes_{K} \bar{K} \stackrel{h}{\rightarrow} F \bar{K} / \bar{R} \stackrel{i}{\rightarrow} \bar{F} / \bar{R}
$$

where $f$ is the isomorphism given by the rule $x \mapsto x \otimes 1$, the maps $g$ and $i$ are inclusion maps, and $h$ is induced by the multiplication map $\mu: F \bigotimes_{K} \bar{K} \rightarrow \bar{F}$. Since $g$ is a split $K-$ module monomorphism, $1 \otimes g$ is a split $R$-module monomorphism. It is enough, therefore, to show that $h$ and $i$ are split $R$-module monomorphisms.

Since $F \bar{K}$ is an $F$-subspace of $\bar{F}$, there is an $F$-subspace $V \subseteq \bar{F}$ such that $\bar{F}=F \bar{K} \oplus V$. As $\bar{R} \subseteq F \bar{K}$ it follows that $\bar{F} / \bar{R}$ is an $R$-module direct sum

$$
\bar{F} / \bar{R}=(F \bar{K} / \bar{R}) \oplus[(V+\bar{R}) / \bar{R}]
$$

and hence that $i$ is a split $R$-module monomorphism.

Finally, consider $h$, which we claim is an isomorphism. There is a commutative diagram with exact rows

$$
\begin{aligned}
& 0 \rightarrow R \bigotimes_{K} \bar{K} \rightarrow F \bigotimes_{K} \bar{K} \rightarrow(F / R) \bigotimes_{K} \rightarrow 0 \\
& \mu^{\prime} \downarrow \quad \mu \quad \downarrow \quad h \quad \downarrow \\
& 0 \rightarrow \bar{R} \rightarrow F \bar{K} \rightarrow F \bar{K} / \bar{R} \rightarrow 0
\end{aligned}
$$

where $\mu^{\prime}$ is the restriction of $\mu$ to $R \bigotimes_{K} \bar{K}$. Note that $\mu$ and $\mu^{\prime}$ are surjective. By Corollary $2.4, \mu$ is an isomorphism, and hence $\mu^{\prime}$ is an isomorphism. Therefore $h$ is an isomorphism, by the Five-Lemma.

Lemma 2.6. Let $R$ be a differential ring and $X$ a left denominator set in the ring $T=$ $R[\theta ; \delta]$. If $\theta \in X$, then $t_{X}\left({ }_{T} R\right)=R$.

Proof. Let $r \in R$. Since $\theta \in X$ there exists $x \in X$ such that $x r \in T \theta$. In addition, $x r-(x \circ r)$ lies in $T \theta$, whence

$$
x \circ r \in T \theta \cap R=0
$$

and so $r \in t_{X}\left({ }_{T} R\right)$. 
Proposition 2.7. Let $R$ be a commutative differential domain with quotient field $F$, and assume that the subfield $K$ of constants of $F$ is contained in $R$. Let $X$ be a left denominator set in the ring $T=R[\theta ; \delta]$ such that $\theta \in X$, and, for all $x \in X$, the $K$-dimension of the solution space

$$
\{r \in R \mid x \circ r=0\}
$$

of $x$ in $R$ equals the order of $x$. Then $F / R$ is isomorphic, as an $R$-module, to a direct summand of $E\left[X^{-1}\right]$ where $E$ is the injective hull of ${ }_{T} R$.

Proof. Let $\bar{F}$ be a differential field extension of $F$ satisfying the conclusion of Proposition 2.1, and let $\bar{K}$ be the subfield of constants of $\bar{F}$.

We first show that $t_{X}\left({ }_{T} \bar{F}\right)=R \bar{K}$. That $t_{X}(\bar{F}) \supseteq R \bar{K}$ follows from Lemma 2.6. To prove the reverse inclusion, it is enough to show that for any $x \in X$, the solution space of $x$ in $\bar{F}$ is contained in $R \bar{K}$. Let the order of $x$ be $n$. By hypothesis, we can choose a $K$-basis $\left\{r_{1}, \ldots, r_{n}\right\}$ for the solution space of $x$ in $R$. By Corollary 2.4, these $r_{i}$ are $\bar{K}$-linearly independent. On the other hand, the solution space of $x$ in $\bar{F}$ has $\bar{K}$-dimension at most $n$, by $[1$, Theorem 1]. Thus

$$
\{\alpha \in \bar{F} \mid x \circ \alpha=0\}=\bar{K} r_{1}+\cdots+\bar{K} r_{n} \subseteq R \bar{K}
$$

as desired.

Now by Proposition 2.2, $\bar{F}\left[X^{-1}\right]=\bar{F} / t_{X}(\bar{F})=\bar{F} / R \bar{K}$, and $E$ may be identified with a $T$-submodule of $\bar{F}$ containing $F$. Since $E$ is an injective $T$-module, it is divisible, whence $E\left[X^{-1}\right]=E / t_{X}(E)$. By Lemma 2.6, $R \subseteq t_{X}(F)$. Now the inclusions $F \subseteq E \subseteq \bar{F}$ induce $R$ module homomorphisms

$$
\rho: F / R \rightarrow E\left[X^{-1}\right] \text { and } \sigma: E\left[X^{-1}\right] \rightarrow \bar{F} / R \bar{K}
$$

whose composition equals the split $R$-module monomorphism $j$ of Proposition 2.5. It follows that $\rho$ is a split $R$-module monomorphism, and so $F / R$ is isomorphic to an $R$ module direct summand of $E\left[X^{-1}\right]$.

Our next aim is to construct examples of $R$ and $X$ satisfying the hypotheses of Proposition 2.7. The method which we shall use to construct $X$ is given by the following result.

Proposition 2.8. Let $T$ be a ring, $c$ a regular element of $T$, and $G$ a group of automorphisms of $T$. Let $X$ be the multiplicatively closed subset of $T$ generated by the set $\{g(c) \mid g \in G\}$. Then $X$ is a left denominator set in $T$ if and only if

$$
\text { For each } t \in T \text { there exists } x \in X \text { such that } x t \in T c \text {. }
$$

Proof. Note that $X$ is closed under the action of $G$, and that $X$ consists of regular elements. In particular, the reversibility condition is trivially satisfied, and so only the Ore condition is of concern.

That $\left(^{*}\right)$ is necessary is clear since $c \in X$. Conversely, suppose that $\left(^{*}\right)$ holds. We 
prove by induction that $X$ satisfies the left Ore condition; namely, given $t \in T$ and $g_{1}, \ldots, g_{n} \in G$ there exist $x_{n} \in X$ and $t_{n} \in T$ such that

$$
x_{n} t=t_{n} g_{n}(c) g_{n-1}(c) \ldots g_{2}(c) g_{1}(c)
$$

For the case $n=1$, condition ( $\left.{ }^{*}\right)$ provides us with elements $x \in X$ and $u \in T$ such that $x g_{1}^{-1}(t)=u c$. Thus $g_{1}(x) t=g_{1}(u) g_{1}(c)$ and hence $x_{1} t=t_{1} g_{1}(c)$ where $x_{1}=g_{1}(x) \in X$ and $t_{1}=g_{1}(u) \in T$. For the inductive step, let $n>1$ and suppose that there exist $x_{n-1} \in X$ and $t_{n-1} \in T$ satisfying

$$
x_{n-1} t=t_{n-1} g_{n-1}(c) g_{n-2}(c) \ldots g_{2}(c) g_{1}(c)
$$

By the case $n=1$ there exist $y \in X$ and $t_{n} \in T$ such that $y t_{n-1}=t_{n} g_{n}(c)$. Therefore, setting $x_{n}=y x_{n-1} \in X$, we obtain

$$
x_{n} t=y x_{n-1} t=t_{n} g_{n}(c) g_{n-1}(c) \ldots g_{2}(c) g_{1}(c),
$$

completing the inductive step.

We shall apply Proposition 2.8 in the case that $T$ is a differential operator ring and $c=\theta$. In order to see that $X$ is a right as well as left denominator set, we use an involution to reverse sides in $T$. Provided $T=R[\theta ; \delta]$ for a commutative differential ring $R$, there is a natural involution ${ }^{*}$ on $T$ such that $\theta^{*}=-\theta$ and $r^{*}=r$ for all $r \in R$.

Proposition 2.9. Let $R$ be a commutative differential ring, $A$ an additive group of constants of $R$, and $T$ the ring $R[\theta ; \delta]$. Let $X$ be the multiplicatively closed subset of $T$ generated by the set $\{\theta+\alpha \mid \alpha \in A\}$. Then the following conditions are equivalent:

(i) $X$ is a left denominator set in $T$.

(ii) $X$ is a right denominator set in $T$.

(iii) For each $r \in R$ there exists $x \in X$ such that $x \circ r=0$.

Proof. (i) $\Leftrightarrow$ (ii): Let $Y$ be the multiplicatively closed set $\{ \pm x \mid x \in X\}$. As $A$ is an additive subgroup of $R$, we see that

$$
Y=Y^{*}=\left\{ \pm z \mid z \in X^{*}\right\}
$$

Now $X$ is a left denominator set if and only if $Y=Y^{*}$ is a left denominator set, if and only if $X^{*}$ is a left denominator set, if and only if $X$ is a right denominator set.

(i) $\Leftrightarrow$ (iii): For each $\alpha \in A$ there is an automorphism $g_{\alpha}$ of $T$ such that $g_{\alpha}(\theta)=\theta+\alpha$ and $g_{\alpha}(r)=r$ for all $r \in R$. The set $X$ is the multiplicatively closed subset of $T$ generated by $\{g(\theta) \mid g \in G\}$ where $G$ is the group $\left\{g_{\alpha} \mid \alpha \in A\right\}$ of automorphisms of $T$. By Proposition 2.8, $X$ is a left denominator set if and only if

For each $t \in T$ there exists $x \in X$ such that $x t \in T \theta$.

Given $t \in T$ and $x \in X$, write $t=r_{0}+r_{1} \theta+\cdots+r_{n} \theta^{n}$ for some $r_{i} \in R$ and observe that

$$
x t-\left(x \circ r_{0}\right) \in T \theta .
$$


Hence, $x t \in T \theta$ for some $x \in X$ if and only if $x \circ r_{0}=0$ for some $x \in X$. Therefore (*) and (iii) are equivalent.

Lemma 2.10. Let $R$ be a commutative differential domain with quotient field $F$. If $R$ is $\delta$-simple then all nonzero constants of $F$ are units of $R$.

Proof. Let $\alpha$ be a nonzero constant of $F$, and let

$$
I=\{r \in R \mid \alpha r \in R\} .
$$

If $r \in I$, then $\alpha \delta(r)=\delta(\alpha r) \in R$ and so $\delta(r) \in I$. Hence, $I$ is a $\delta$-ideal of $R$, and we note that $I$ is nonzero. It follows, by the $\delta$-simplicity of $R$, that $I=R$, whence $\alpha \in R$. Now $\alpha R$ is a nonzero $\delta$-deal of $R$, and so $\alpha R=R$. Thus $\alpha$ must be a unit of $R$.

Proposition 2.11. Let $K$ be a field of characteristic zero, let $n$ be a positive integer, and assume that $K$ contains $n$ elements $\alpha_{1}, \ldots, \alpha_{n}$ that are $\mathbb{Q}$-linearly independent. Let $A$ denote the additive group $\mathbb{Z} \alpha_{1}+\cdots+\mathbb{Z} \alpha_{n}$. Let $F$ be a rational function field $K\left(x_{0}, x_{1}, \ldots, x_{n}\right)$ with the $x_{i}$ algebraically independent over $K$, and let $\delta$ be the $K$-linear derivation on $F$ such that $\delta\left(x_{0}\right)=1$ and $\delta\left(x_{i}\right)=\alpha_{i} x_{i}$ for $i>0$. Let $R$ be the differential subring $K\left[x_{0}, x_{1}^{ \pm 1}, \ldots, x_{n}^{ \pm 1}\right]$ of $F$, and let $X$ be the multiplicatively closed subset of the ring $T=R[\theta ; \delta]$ generated by the set $\{\theta+\alpha \mid \alpha \in A\}$.

(i) $R$ is $\delta$-simple.

(ii) The subfield of constants of $F$ is $K$.

(iii) $X$ is a left and right denominator set in $T$.

(iv) For all $x \in X$, the $K$-dimension of the solution space of $x$ in $R$ equals the order of $x$.

Proof. (i) The differential subring $S=K\left[x_{1}^{ \pm 1}, \ldots, x_{n}^{ \pm 1}\right]$ of $R$ is $\delta$-simple by [9, Theorem 2.1]. Note that an element $s \in S$ cannot satisfy $\delta(s) \in K$ unless $s \in K$, in which case $\delta(s)=0$.

Let $I$ be a nonzero $\delta$-ideal in $R=S\left[x_{0}\right]$, and let $m$ be the minimal degree in $x_{0}$ of nonzero elements of $I$. The set

$$
J=\left\{s_{m} \in S \mid s_{m} x_{0}^{m}+s_{m-1} x_{0}^{m-1}+\cdots+s_{0} \in I \text { for some } s_{m-1}, \ldots, s_{0} \in S\right\}
$$

is a nonzero $\delta$-ideal of $S$, and so $J=S$. Hence, there exist elements $s_{m-1}, \ldots, s_{0}$ in $S$ such that the element

$$
y=x_{0}^{m}+s_{m-1} x_{0}^{m-1}+\cdots+s_{1} x_{0}+s_{0}
$$

lies in $I$. But the element

$$
\delta(y)=\left(m+\delta\left(s_{m-1}\right)\right) x_{0}^{m-1}+\left[\text { terms of degree } \leqq m-2 \text { in } x_{0}\right]
$$

also lies in $I$, whence $\delta(y)=0$, by the minimality of $m$. As a result, $\delta\left(s_{m-1}\right)=-m$, and so $m=0$. Thus $I \cap S \neq 0$, and therefore, since $S$ is $\delta$-simple, $I \cap S=S$ and $I=R$. 
(ii) Any unit $u$ of $R$ has the form

$$
u=\beta x_{1}^{m(1)} x_{2}^{m(2)} \ldots x_{n}^{m(n)}
$$

for some nonzero $\beta \in K$ and some integers $m(i)$, and

$$
\delta(u)=\left[m(1) \alpha_{1}+m(2) \alpha_{2}+\cdots+m(n) \alpha_{n}\right] u .
$$

If $u \notin K$, then at least one $m(i) \neq 0$ and $\delta(u) \neq 0$ by the $\mathbb{Q}$-linear independence of the $\alpha_{i}$. Hence, (ii) follows from (i) and Lemma 2.10.

(iii) By Proposition 2.9 it is enough to show that for each $r \in R$ there exists $z \in X$ such that $z \circ r=0$. First suppose that

$$
r=x_{0}^{m(0)} x_{1}^{m(1)} \ldots x_{n}^{m(n)}
$$

for some integers $m(i)$ with $m(0) \geqq 0$. If

$$
y=\theta-m(1) \alpha_{1}-\cdots-m(n) \alpha_{n},
$$

then $y^{m(0)+1} \in X$ and $y^{m(0)+1} \circ r=0$. Since $R$ is spanned over $K$ by monomials of the above form, and since $X$ is commutative, the desired condition follows.

(iv) Let $x \in X$ be of order $k$. Then $x$ can be written in the form

$$
x=\left(\theta-\beta_{1}\right)^{m(1)}\left(\theta-\beta_{2}\right)^{m(2)} \ldots\left(\theta-\beta_{t}\right)^{m(t)}
$$

where the $\beta_{j}$ are distinct elements of $A$, the $m(j)$ are positive integers, and $\sum m(j)=k$. There are integers $p(j, i)$, for $j=1, \ldots, t$ and $i=1, \ldots, n$, such that

$$
\beta_{j}=\sum_{i=1}^{n} p(j, i) \alpha_{i}
$$

for each $j$. Set $y_{j}=x_{1}^{p(j, 1)} x_{2}^{p(j, 2)} \cdots x_{n}^{p(j, n)}$ for $j=1, \ldots, t$. It is a routine calculation to check that the set

$$
\left\{x_{0}^{m} y_{j} \mid j=1, \ldots, t \text { and } m=0, \ldots, m(j)-1\right\}
$$

is a $K$-linearly independent set of $k$ elements of the solution space of $x$ in $R$. By $[1$, Theorem 1], this solution space has dimension exactly $k$.

We are now in a position to construct our example.

Theorem 2.12. Let $n$ be a positive integer. There exist a simple noetherian domain T, a left and right denominator set $X$ in $T$, and an injective left T-module $E$ such that

$$
\text { K.dim. }(T)=\operatorname{gl} \cdot \operatorname{dim} .(T)=n+1
$$

and inj.dim. $\left(E\left[X^{-1}\right]\right)=n$. Moreover, $E$ is the injective hull of a simple left T-module. 
Proof. Choose a field $K$ of characteristic zero which has $\mathbb{Q}$-dimension at least $n$, and let $R, T, X$ be as in Proposition 2.11. Then $R$ is a $\delta$-simple, commutative, noetherian, regular, differential domain of Krull and global dimension $n+1$. By [2, Theorem 6.3], ${ }_{R} R$ has injective dimension $n+1$. If $F$ is the quotient field of $R$, then since ${ }_{R} F$ is injective, ${ }_{R}(F / R)$ must have injective dimension $n$.

Note from the $\delta$-simplicity of $R$ that $R$ is a simple left $T$-module. As $R$ is a $\delta$-simple noetherian $\mathbb{Q}$-algebra and $\delta \neq 0$, the domain $T$ is a simple noetherian ring [4, Proposition 3.1, Theorem 3.2]. Consequently, $T$ has Krull and global dimension $n+1$, by [7, Theorems 2.6 and 3.2$]$.

Let $E$ be the injective hull of ${ }_{T} R$. By Propositions 2.11 and 2.7, $F / R$ is isomorphic, as an $R$-module, to a direct summand of $E\left[X^{-1}\right]$. Hence,

$$
\text { inj.dim. }\left({ }_{R} E\left[X^{-1}\right]\right) \geqq \operatorname{inj} \cdot \operatorname{dim} .\left({ }_{R}(F / R)\right)=n .
$$

Since $T$ is flat as a right $R$-module, we conclude using [8, Theorem IV.12.5] that

$$
\text { inj.dim. }\left({ }_{T} E\left[X^{-1}\right]\right) \geqq \operatorname{inj} \cdot \operatorname{dim} .\left({ }_{R} E\left[X^{-1}\right]\right) \geqq n .
$$

Recall that $E\left[X^{-1}\right] \cong E / t_{X}(E)$. If ${ }_{T} E\left[X^{-1}\right]$ had injective dimension $n+1$, then $t_{X}(E)$ would have injective dimension $n+2$, which is impossible. Therefore inj.dim. $\left({ }_{T} E\left[X^{-1}\right]\right)=n$.

\section{REFERENCES}

1. S. A. Amirsur, A generalization of a theorem on linear differential equations, Bull. Amer. Math. Soc. 54 (1948), 937-941.

2. H. BAss, Injective dimension in noetherian rings, Trans. Amer. Math. Soc. 102 (1962), 18-29.

3. A. W. Chatrers and C. R. Hajarnavis, Rings with Chain Conditions (Pitman, Boston, 1980).

4. J. H. Cozzens and C. FAITH, Simple Noetherian Rings (Cambridge University Press, Cambridge, 1975).

5. E. C. DADE, Localization of injective modules, J. Algebra 69 (1981), 416-425.

6. K. R. Goodearl, Global dimension of differential operator rings, Proc. Amer. Math. Soc. 45 (1974), 315-322.

7. R. HART, Krull dimension and global dimension of simple Ore-extensions, Math. Zeitschrift 121 (1971), 341-345.

8. P. J. Hilton and U. Stammbach, A Course in Homological Algebra (Springer-Verlag, Berlin, 1971).

9. J. C. MCConnelL, Representations of solvable Lie algebras. V. On the Gelfand-Kirillov dimension of simple modules, J. Algebra 76 (1982), 489-493. 1972).

10. D. W. Sharpe and P. Vámos, Injective Modules (Cambridge University Press, Cambridge,

11. B. Stenstrom, Rings of Quotients (Springer-Verlag, Berlin, 1975).

Department of Mathematics

UNIVERSITY OF UTAH

Salt Lake City, Utah 84112, USA
Department of Pure Mathematics UNIVERSITY OF SHEFFIELD SHEFFIELD S3 7RH, ENGLAND 